\title{
Applying simulation modeling techniques for the design and assessment of control software
}

\author{
Il. Astinov, N. Todorov \\ Laboratory 'Simulation Modeling in Industry' (SMI) \\ MTF, TMMM, Technical University - Sofia (VMEI), 1756 Sofia, \\ BULGARIA, Tel: (+359 2) 636 3784, FAX: (+359 2) 683 478, \\ E-mail: ila@vmei.acad.bg
}

\begin{abstract}
The paper presents a non-traditional application of simulation modeling techniques as a decision aiding tool in determining the effectiveness of a control software algorithm in manufacturing systems. Normally software engineering methods and techniques would bring the development of a control software module to a level where certain predesigned functionality is achieved. In many cases however, such functionally will not guarantee a reliable operation of the overall system. Additionally, stochastic factors such as the randomness of workpiece arrival intervals, processing times, breakdowns, which influence the operation of the real system are not taken into account. An application in using simulation modeling techniques to evaluate the effectiveness of different control algorithm designs and structures, prior to their implementation in real systems is presented in this paper.
\end{abstract}

\section{Keywords}

Modeling, simulation, decision making, control technology

\section{INTRODUCTION}

Control software plays a significant role in the effectiveness of modern manufacturing systems. Typically control software is designed to run on general purpose or specialized computers. It would operate on the principles outlined below.

- Analog or digital signal will be fed to the computer running the control software. These signals contain information reporting the state of the system being controlled. 
- The control software will process the incoming information and according to the control algorithm will react (if required) with appropriate feedback information.

- The feedback information is translated by appropriate hardware to follow-up actions on behalf of the system.

Figure 1 is an illustration of these principles which shows the information streams coming from the system. These streams being processed by the control algorithm and then relevant control information flowing back to the system as a product of the control algorithm.

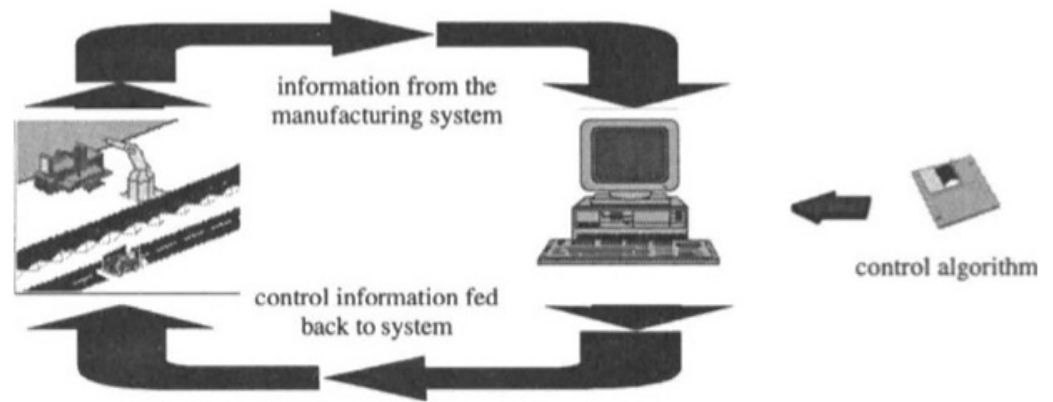

Figure 1 Loops of information streams in manufacturing systems.

A well designed and error free code would reduce time for manufacturing, increase the system reliability yet contribute to a lower cost of the end product. The paper presents the experience of the authors in utilizing simulation modeling techniques aiming to develop effective new or improve existing control algorithms.

\section{PROBLEM DEFINITION AND SOLUTION PROPOSED}

\subsection{Problem definition}

A traditional approach in the design and implementation of such software is to utilize software engineering methodologies in developing the algorithm and code. Later on the control software is tested on the reat system i.e. on the manufacturing cell, on the AGV or in the warehouse (see Figure 1). Apart from the general advantage that the performance of the real system is being observed, this approach has the following disadvantages.

- Tests generally are limited in time as manufacturing systems are to be put in operation within a strict deadline.

- Experimenting with the real system is obviously an expensive and in many cases could be a dangerous exercise.

- The majority of the stochastic factors like the randomness of workpiece arrival intervals, processing times, breakdowns, which influence the operation of the overall system can rarely be taken into account. 


\subsection{Solution proposed}

In order to address extent the mentioned disadvantages, a solution to the problem was adopted, having the outline given below.

- Create a model of the manufacturing system using appropriate and familiar simulation software. This could be any general purpose software like SLAMSYSTEM or a simulator like FACTOR/AIM.

- Design alternatives to the control algorithm for the components of the system that are to be controlled. If necessary, produce code for each alternative in a language, that can be linked to the simulation software. Most simulation systems have an open architecture structure allowing user inserts, written in high level languages, such as FORTRAN 77 or C++ to be linked to the main executable file, gain access to the data structures and manipulate them.

- Create scenarios each of which will utilize a version of the control algorithm. Define clear performance measures of the system. Define and perform experiments with the scenarios of the model.

- Compare the values of the performance measures estimated as results of the simulation runs and take appropriate decisions for the choice of the most effective version of the control algorithm.

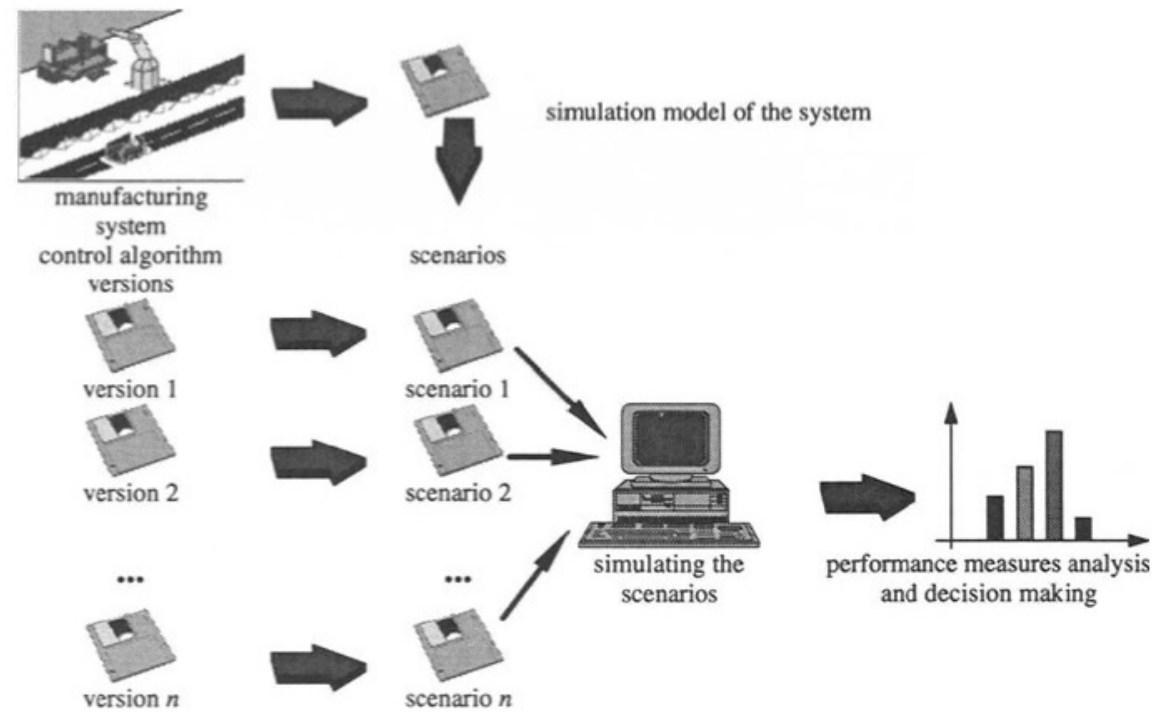

Figure 2 Concept of the solution proposed.

Figure 2 illustrates the concept of the solution proposed. By this means simulation methodology and more particular discrete event simulation is utilized to a full extent as a powerful tool in modeling manufacturing systems (Astinov 1990, Pritsker 1986). Yet in this particular case (Figure 2) the effectiveness of the control algorithm over the identified 
performance measures of the system is being studied, rather than the system as a whole. The following section describes a case study, illustrating the effect of the presented approach.

\section{CASE STUDY - THE KILN FACILITY}

\subsection{Components and their purpose in the facility}

The case study relates to a kiln facility, typical for companies manufacturing ceramic products such as tableware (cups, plates), bathroom and toilet equipment. An example of the configuration of such facility is given in Figure 3.

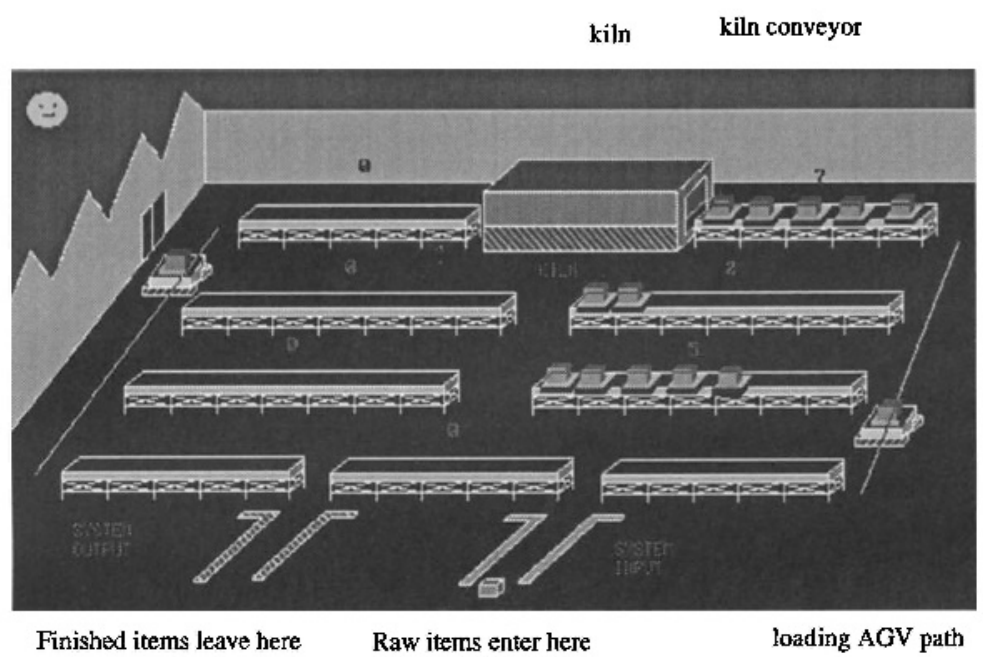

Figure 3 Typical configuration of a kiln facility.

Items to be fired in the kiln arrive at random intervals in the input of the system. They are placed on pallets in the pallet loading station and afterwards the loading AGV would distribute them over the input conveyors. The distribution pattern depends on the control algorithm incorporated in the AGV controller. Once placed on the conveyor that feeds the kiln, the items are transported at a rate, that will allow them to be fired for the appropriate length of time. The conveyors after the kiln are used to hold the pallets with fired items during the night shift, as the kiln operates on a 24 hour basis, and during the night pallets with fired items are not removed from the system.

\subsection{Versions of the AGV control algorithm}

The present case study concerns an existing kiln facility, which had a particular control algorithm for the loading AGV implemented. From an operational point of view, the algorithm 
performed the control functions exactly as it was designed. The principle structure of the algorithm is outlined in Figure 4 (a).

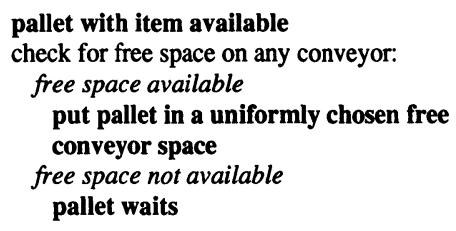

(a) Outline of the existing control algorithm.

\author{
pallet with item available \\ check for free space on kiln conveyor: \\ free space on kiln conveyor available \\ put pallet on kiln conveyor \\ free space on kiln conveyor not available \\ check for free space on any other conveyor: \\ free space available \\ put pallet in a uniformly chosen free \\ conveyor space \\ free space not available \\ pallet waits
}

(b) Outline of the proposed improvement of the control algorithm.

Figure 4 Structures of existing and proposed AGV control algorithms.

It was thougt that the utilization of the kiln could be improved by giving priority to the kiln conveyor when a pallet with an item arrives. The outline of the proposed improvement is given on Figure 4 (b). The decision whether such a modification would be worthwhile was difficult to make because of the facts given below.

- The kiln facility as a system had stochastic properties, as the arrivals of parts at the system's input were in random intervals of time.

- The speed pallets were transported through the kiln was so low that the impact on giving priority to the kiln conveyor (whether positive or negative) was not very clear.

- If such change in the control algorithm proved to be ineffective this could lead to significant losses in terms of production quantities and energy. Such risk was not accepted by the management of the company.

\subsection{The solution derived}

The methodology described in Section 2 was employed in order to give a justified answer to the problem. A model of the kiln facility was developed using the SLAMSYSTEM general purpose simulation software (SLAMSYSTEM 1993). Appropriate code was developed for both versions of the AGV control algorithm.

Face validity and expert assessment were employed to validate the operation of the model, using the code of the existing AGV control software. Two scenarios were created each using one of the versions of the control algorithm. For both scenarios computer animation was produced, illustrating the dynamic behavior of the kiln facility. Separate frames of this output are given in Figure 5. 


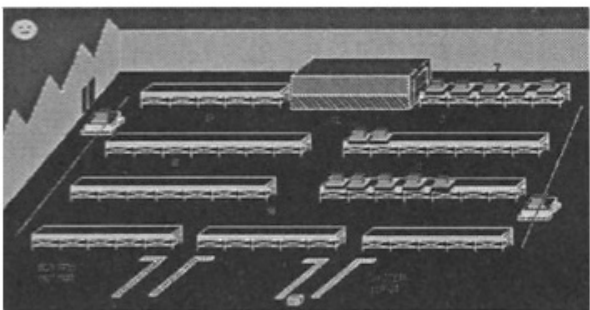

(a) Day shift view

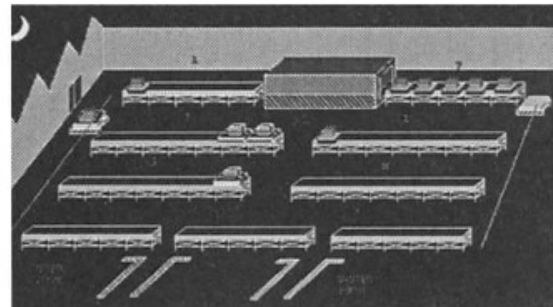

(b) Night shift view

Figure 5 Computer animation frames illustrating the dynamic system behavior.

The performance measure identified was the system throughput for 24 hours on the assumption that no other conditions of operation will be changed except the AGV control. The values of the throughput of the kiln facility estimated by the simulation runs are compared and illustrated on the bar graph given in Figure 6.

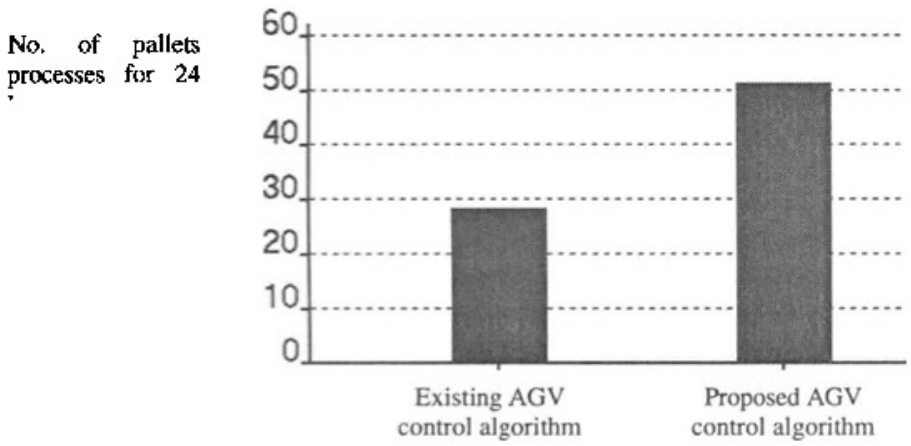

Figure 6 Comparison of the throughput of the kiln facility utilizing different AGV control algorithms.

As it is seen from Figure 6, the implementation of the control algorithm with the structure given on Figure 4(b) will lead to an increase of the kiln facility throughput with an average of 22 pallets i.e. $39 \%$ in 24 hours. This result is a strong proof of the increased effectiveness of the new algorithm and suggests that it would be worthwhile to proceed to implementation.

This approach was extensively utilized in two other significant projects. The first one related to power stations using combined multiple renewable energy sources (wind, solar irradiation, batteries and diesel units). The project was sponsored by the Directorate General for Science and Technologies of the European Union under the PECO Scheme (Project ERBJOU2CT920245). Two major control approaches and related algorithms of the power station were studied. Performance measures included diesel fuel consumption, energy produced by renewables, total energy from renewables dumped in the dump load. These were evaluated through separate simulation runs. The system was strongly influenced by stochastic factors, such as power consumption, wind speed, solar irradiation and temperature. Values of 
each of these factors vary over time in a random manner (Astinov, 1994; Astinov Ivanova, Pavlov et al 1995).

The second project concerned the improvement of the through put of a major crossroad in Sofia, controlled by traffic lights (Astinov, Ivanova, Stoichkov et al, 1995). This project is currently under development in collaboration with the company involved in the installation and maintenance of urban traffic control systems in Sofia. The crossroad as a system is also stochastic, as car arrival intervals from each direction are random. The component in the system that can be modified is the control algorithm changing light signals for each direction. For the test case an improvement of an average of $18 \%$ was estimated for one of the control algorithms.

\section{CONCLUSIONS}

The following conclusions can be drawn, based on the results achieved so far:

- As described in Section 3.2, a version of the control algorithm may be designed according to a specified functionality. It may also be developed in appropriate code and be error free. It may operate exactly as the designers wanted it to operate. However, this is not a guarantee that the control functionality incorporated in the particular algorithm will assure good overall system performance. The major advantage of the reported approach is that it (the approach) allows the developers of control software to verify not only the proper operation and correct structure of the control algorithm, but to evaluate the effectiveness of the algorithm over the operation of the overall system based on redefined performance measures.

- The proposed approach eliminates the disadvantages of the development and implementation of control software given in Section 2.1. Moreover, as many versions of control algorithms as needed may be developed and studied, thus aiding the decision making process towards a rational and validated choice of suitable control algorithm functionality.

- Simulation runs can be performed for a long duration of the operation of the system, taking into account the stochastic properties of system's behavior (if available). Thus, the estimates of performance measure values will be statistically justified and correct.

- Computer animation of the system's operation is an additional tool in this approach, allowing experts to gain a realistic view of the control algorithm's influence to the overall operation of the system.

\section{ACKNOWLEDGMENTS}

The developments and results reported in this paper are part of the activities of the HC\&M project ERBCIPDCT940023 sponsored under the PECO scheme of the European Union.

The activities on the simulation of combined multiple renewable systems, mentioned in the present paper were part of the activities of the ERBJOU2CT920245 project sponsored under the PECO scheme of the European Union.

Words of thank are to be said to Sonia Hristova, Hristo Dinov and Dinitar Cerov all from Traffic Signs Ltd. - Sofia for their assistance and collaboration in the crossroad project. 
Finally the authors would like to express their appreciation to Zlatka Tchkarova, Wladimir Simeonov, Blagovest Stoichkov and the CMRES team - all students at the laboratory for Simulation Modeling in Industry for their valuable contributions in the mentioned projects.

\section{REFERENCES}

Astinov, Il. (1990) Simulation modeling of machine tools and systems. PhD thesis. Technical University Sofia, Sofia.

Astinov I.L., Bopp G., Consoli A., Lalas D.P., Morgana B., Wrixon G.T. (1994) Combined Multiple Renewable Energy Sources System Simulator Facility. Proceedings of the European Wind Energy Association Conference and Exhibition (EWEC'94), Thessaloniki

Astinov Il., Ivanova D., Pavlov P, Virgili A., Leotta, Nocera V., Bopp G., Rehm M.. A.(1995); Simulating power stations driven by combined multiple renewable energy sources. International Symposium SIELA'95, Volume II, 183-188

Astinov Il., Ivanova D., Stoichkov B., Simeonov W. (1995) Simulation modeling in the control of traffic flow. Proceedings of the III National Scientific Conference "Automation in Engineering and machine tool building", Volume 1, 73-76

Pritsker, A.A.B. (1986) Applications of simulation. Introduction to simulation and SLAM II. Halsted Press, John Wiley \& Sons, New York, Chichester, Brisbane.

SLAMSYSTEM User's Guide (1993) Pritsker Corporation

\section{BIOGRAPHY}

Prof. Ilario Astinov has an MSc degree in production engineering and $\mathrm{PhD}$ degree in systems simulation from the Technical University - Sofia. Has over 25 publications in national and international forums. He has specialized in discrete event simulation at Purdue University (USA), Pritsker Corporation (USA), Staffordshire University (UK) and Cranfield University (UK). He is head of the laboratory for Simulation Modeling in Industry at the Technical University - Sofia and a board member of the UNIDO/DP/BUL/006/96 project. He is Deputy Coordinator of three international academic projects under the EU TEMPUS scheme and Associate Contractor to three international research projects under the EU PECO scheme involving partners from $8 \mathrm{EU}$ countries.

Prof. Nikola Todorov has an MSc and $\mathrm{PhD}$ degree in the field of mechanical and production engineering. He has over 40 publications in national and international conferences and magazines. He is head of the laboratory "CAD/CAM and FMS technologies" at the Technical University - Sofia. For over 15 years he was the head of the department of CAD/CAM applications at the Institute of Machine Tools and Systems - Sofia. He is a board member of the UNIDO/DP/BUL/006/96 project. 